\title{
ADVANCED METHOD FOR QoE EVALUATION AND IMPROVEMENT IN MODERN CELLULAR NETWORKS
}

\author{
Roman Odarchenko ${ }^{1,2}$ \\ ${ }^{1}$ Telecommunication systems department \\ National aviation university \\ Kyiv, Ukraine \\ ${ }^{2}$ Bundleslab KFT \\ Budapest, Hungary \\ odarchenko.r.s@ukr.net
}

Anastasiia Abakumova

Telecommunication systems department

National aviation university

Kyiv, Ukraine

nastia.abakumova@gmail.com

\author{
Serhii Bondar \\ International Research and Training Center for \\ Information Technologies and Systems of the National \\ Academy of Sciences (NAS) of Ukraine and Ministry \\ of Education and Science (MES) of Ukraine \\ orangearrows@bigmir.net \\ Yuri Bogachuk \\ International Research and Training Center for \\ Information Technologies and Systems of the National \\ Academy of Sciences (NAS) of Ukraine and Ministry \\ of Education and Science (MES) of Ukraine \\ dep185@irtc.org.ua
}

\author{
Kundyz Maratkyzy \\ Information Systems \\ Satbayev University \\ Almaty, Kazakhstan \\ kundyz.maratkyzy@mail.ru
}

\begin{abstract}
QoE is almost the most important parameter, estimating which it is possible to determine user experience and compare it with users' expectations. Respective method based on the principles of machine learning has been developed to assess and optimize the state of the network in order to improve the quality of service provision to users by cellular operators using the model of service quality control system. Proposed method is a very effective instrument for request formation from users, network status monitoring, service providing quality control integral marks modelling and their optimization, search of degradation reasons and reporting ranging.
\end{abstract}

Keywords-5G, 5G-TOURS, QoE, KPI, QoS, evaluation.

\section{INTRODUCTION}

Permanent and balanced development of the communication branch is inseparably connected to the increasing of the subscribers and provided services. For the cellular service market such tendency is specifically significant - from the one hand, there is a huge subscriber interest for the new service obtaining, and, from the other hand, high concurrence pushing providers to bring in some brand new suggestions on the connection market permanently.

Modern cellular communication service market development level, service providing devices and methods quality changes, information volume and multidimensionality level increasing that circulates at cellular communication networks are advanced the problem of service quality evaluation system modernization that defines in many ways the cellular communication concurrent advantages realization rate.

In the interest of competitiveness saving, service providers are forward their basic efforts to the quality increasing and subscriber network service duration time extension by the implementation of special loyalty program implementation, effective promotion system of existent and brand new additional cellular communication services.

Supporting of the required level of customer satisfaction in high-quality services has complex character and presumes the system of organizationally-technical and socio-economic events for reduction of the achieved service quality level relatively to existing, incipient or prescribed needs of subscribers.

On the basis of the foregoing, providers are need to adopt the subscriber-oriented service model for the data and voice service operation quality increasing and to attract more subscribers to new services, creating the concurrent advantage by the same token. The method is expected to be helpful for the cellular communication providers in the way of people complaint quantity reducing, QoE improvement and enhancement of the subscribers loyalty, especially of the subscribers with high cost (key subscribers).

\section{BACKGROWND WORK}

Even though in [1] the whole process of evaluation has been presented, it is not including the influence of different subscriber factors for QoE. Prediction model in [1] was optimized in the development [2], taking into account the 
time of user scrolling, pause time and some other different factors. However, the precise formula has not been obtained at the document, but it was just proved that including factors of subscriber behavior could increase certainty of QoE prediction models. The supposition about the training connection between an application network traffic and each respective QoE-mark that has been represented in [3], is the new concept.

Such concept works in the following way: firstly, control system data is gathering, where provider has as traffic of some service as QoE metrics of the service. After that machine-aided learning algorithm is using for dependency between these values function learning [4]. Such method fits as for operator for the quality support as for numerous network services providers that allows them to proceed the analysis of different anomalies in provided services. Moreover, it can help in bad quality fundamental reason diagnostics by the way of significant network functions definition. Nevertheless, such method has limits because of provided data inexactness, bad users behavior modelling in the needed service and also different learning methods could have influence differently on the analysis quality. More complex learning methods could increase certainty of QoE models even more.

On the basis of the Q-learning algorithm, the HASclient has been proposed in [5], that can configure dynamically, respectively to the network status. Considering the necessary speed for video, the network volume and repeated buffering, the equation MOS receiving in [3] has been proposed. According to the supposition about existence of the simple linear reflection between MOS and PSNR (Peak Signal to Noise Ratio) in [6], and on the basis of the profile length and level of the replicated video quality, predicted MOS is represented in [7].

\section{PROBLEM STATEMENT}

Nowadays traditional communication services proposed by cellular providers are turning obsolete and their places are being taken by [8-9] services, that provide wide service variety: streaming and interactive services, message and data exchange services. In the comparison to basic telecommunication services, new services are need to have cellular provider support. So, it is necessary to use mathematical models and methods that would help to provide required quality of such services.

So, the aim of this work is the cellular communication providers service quality evaluation method modernization.

For the achieving of prescribed aim in this work complex of following interrelated problems has been solved: (i) modern cellular communication systems have been analyzed for the point of structural system definition that are responsible for the service providing quality control and their drawbacks determination; (ii) basic key criteria of cellular network effectiveness that are having significant influence on the user service providing quality by the cellular provider have been defined; (iii) dependence between cellular network parameters definition method for service providing quality evaluation has been developed; (iv) service quality control system and cellular network status evaluation using machine-aided learning model has been developed; (v) cellular network status monitoring and optimization system for service providing quality control has been modeled.

\section{QOE IMPROVEMENT METHOD}

For the evaluation and optimization of the network status for the user service providing quality improving by the cellular network providers with employment of the service quality control system, the respective method based on the machine-aided principles that realizes in three stages has been developed. Represented method can be implemented to services of each type that is provided cellular communication provider.

\section{Stage 1 - Claim analysis}

\section{Step 1. User request arrival}

Using twenty-four-hour chatbot for client service, user can receive immediate answers, to solve questions with machine-aided learning service automatization in the request service domain and respective systems, and also send an application.

Firstly, the process of subscriber identification takes place, in other words if he is a client of some concrete cellular communication provider, is his number a contract or prepaid, etc. Furthermore, chatbot forms an electronic claim on the basis of data that is needed for comprehension of the problem and complaint reasons:

- $\quad$ communication type (voice or data);

- $\quad$ communication standard $(2 \mathrm{G}, 3 \mathrm{G}, 4 \mathrm{G})$;

- $\quad$ service type;

- $\quad$ location place (address or geolocation);

- local appeal place (on the street, in house, at basement, etc.).

Cellular communication service types are forming and choosing with respect to [10] and ETSI TS 102 250-2 [11], and data transfer service types and Internet availability with respect to $[10,12]$.

\section{Step 2. Claim content recognition}

After the claim formation by user, chatbot sends it to the network, where by the help of machine-aided learning the claim content analysis process takes place. If the claim is correct, user would receive message about the claim receiving for processing. If it is not, the request for moderation accomplishment would be assigned to the corporate staff. The staff checks the claim for its filling validity. If there are some faults, it would be corrected by staff and that corrected claim would be resending to the network for admission to processing. At the situation, when the claim is filled correctly but anyways has been redirected to staff, "machine" conceive such claim as brand new, so it is necessary to moderate machine-aided learning.

\section{Step 3. Request proceeding}

On this step data reading and processing from the claim is achieving. The result of such process is the definition of primary information for problem solving, specifically the system obtains information about the problem type, connection standard, location area, etc.

Stage 2 - KPI settings analysis
Step 1.
Primary
definition 
After the machine obtains the information about, so to speak, origin of the problem, it forms series of KPI settings (primary), in other words, such settings are used for that problem working analysis in general.
Cellular network KPI by the categories [13] are demonstrating on the following Fig. 1.

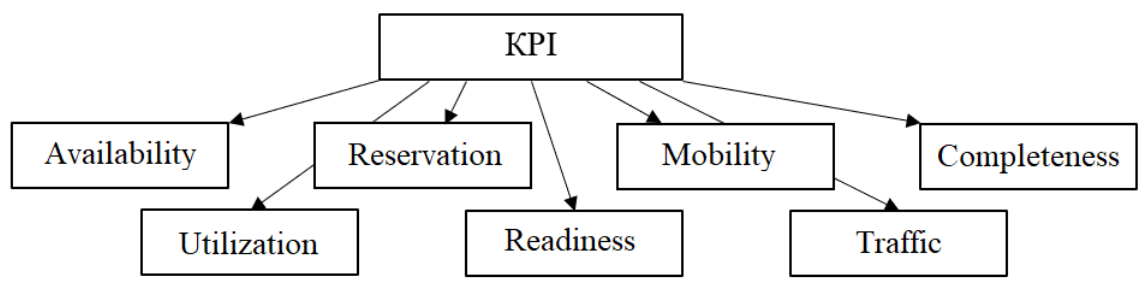

Fig.1. KPI scheme by categories

Let's consider each cellular network KPI category in more detail.

KPI of availability. KPI of availability measurement helps the provider of the cellular network receive the information if it is possible to have the access to service, that has been requested by user with some special accesslevels under some conditions. E-UTRAN service defines as EPS (Evolved Packet System) or E-RAB (E-UTRAN Radio Access Bearer), RRC (Radio Resource Control) and SAE (System Architecture Evolution), that are KPI availability basic procedures KPI [13-14].

$K P I$ of reservation. If final users are often interrupted during the using of service or if the service ceases during the usage, providers have troubles with charging a fee for a time during that service is not providing. KPI for reservation measuring system capability for supporting of consequential repetitive usage and to accomplish prescripted functions. Reservation of the final utilization spans much more wide area than E-UTRAN, in other words KPI of prescripted area are limited by parts upon EUTRAN has control [13-14].

KPI of mobility. Mobility is the fundamental functionality that provides ceaseless servicing of users that are moving through the network. KPI of mobility are dealing with handovers straightforwardly. Measuring includes as intra-UTRAN as inter-RAT (Radio Access Technology) handovers. Measurements are proceeding on the cell and cluster levels. Handovers are defining active UE connection sending from one cell to another. Each handover in LTE is hard so the connection between UE and RAN interrupts temporarily during handovers [14-15].

KPI of completeness. Completeness marks are demonstrating E-UTRAN influence on service quality that are being provided to customer. Service KPI of completeness are calculating on the cell or cluster levels. It is necessary to take into account that cluster level KPI is an aggregation of cell level counters $[13,15]$.

KPI of utilization. KPI of utilization is using for evaluation of system capability for satisfaction of traffic needs $[22,21]$.

KPI of availability. KPI of availability are measuring the percent of time during that cell is available, specifically when eNodeB can provide EPS services [15].

KPI of traffic. KPI of traffic are using for the purpose of traffic volume measurement on the LTE network radio access. KPI of traffic are classified on the traffic type basis: radio channels, downline connection traffic volume and upline connection traffic volume [16].

\section{Step 2. Comparison to the calibration values}

On the given step, stream values of series from primary parameters comperes to the calibration values in SLA contracts, characterizing the actual service status. If each parameter is in the normal limits, it is regarded that problem is on the user side, for example, an obsolete phone model or an old SIM-card, and claim closes. Otherwise, parameter(s) that are not corresponded to the calibration values or exceed the normal limits are recorded and fault messages are generating.

\section{Stage 3 - Optimization}

\section{Step 1. Secondary parameters determination}

Before the problem parameter optimization proceeding, choosing of such secondary KPI parameters that are having the most significant influence on its value need to be done. For definition of the functional dependence between network parameters the method of Hermith cubic spline that is described in the following section would be used.

This process is the kernel of system. On the basis of received KPI values, the service quality coefficient (KQI) calculation accomplishes and their uninterrupted monitoring in real time mode is provided.

The calculation of service quality coefficients proceeds with application of service quality parameter results. Herewith, it is deemed that user knows how to use the cellular gadget and the service. It is necessary to say that cellular instrument working ability parameters evaluation is not taking place. During the cellular communication quality parameter measurement, it is assumed that service is in the stage of readiness, usage of it is not prohibited, cellular instrument has right settings and routing was done without any error.

\section{Step 2. Additional information analysis}

As additional information could be considered: (i) the time of day; (ii) weather conditions; (iii) BS (base station) workload; (iv) mass event organizing.

\section{Step 3. Decision making}

In this step, the system decides on the type of network optimization: software or hardware. And after this, the optimization process is taking place.

\section{QOE IMPROVEMENT METHOD}

For the achievement of the service providing quality improving mission it is needed to derive the functional dependency between the network parameters, in other words, it is needed to define the influence of key performance indicator (KPI) on key quality indicator (KQI) [17-19]. 


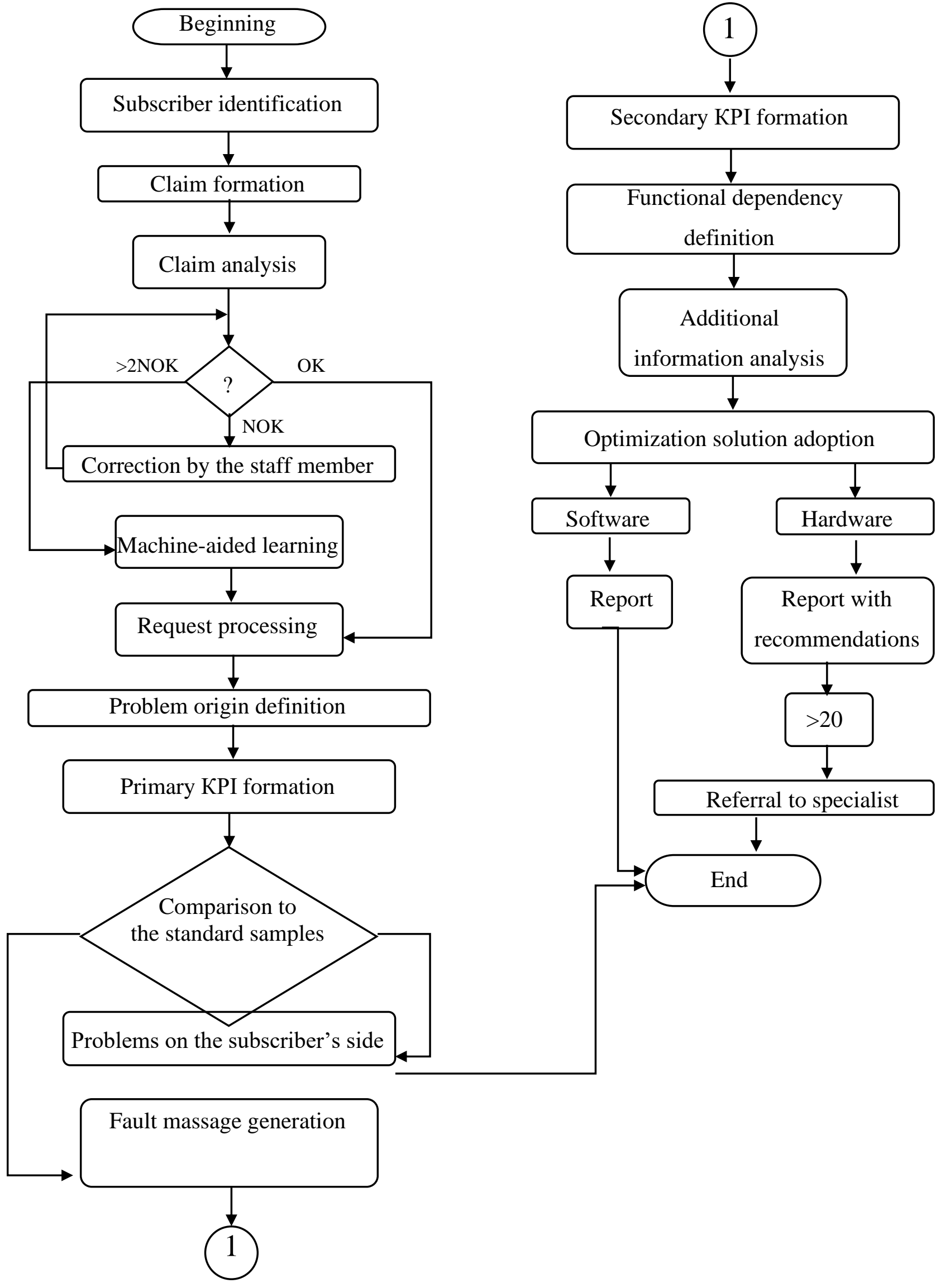

Fig. 2. Cellular network condition evaluation method working algorithm 
The problem of functional dependency definition between the network parameters KPI and KQI can be solved by method of spline usage.

There are different ways to build splines on the basis of their types [20-22]. But the most interest here are local Hermith cubic splines (KEC). They differ from global by the calculation simplicity, at the expense of that they provide high operation speed during the calculation that at another point is very important for work in real time mode during the proceeding of large data arrays.

For the construction of KEC merging nodes are spotting in such a way that spline on the whole interval satisfies least square method (LSM) conditions:

$\sum_{i=1}^{N}\left[S p_{i}-y_{i}\right]^{2}=\min$,

where $N$ - counting quantity on the monitored interval;

$i-$ serial number of counting;

$S p_{i-\text { spline; }}$

$y_{i}-$ input data.

After the definition of the formula (1) we can assure that total squared error of the approximating spline $y_{i}$ from the function would be minimal.

Further, let us calculate the planning matrix $P$ that has block-diagonal structure as a result of local features of the function (fig. 3).

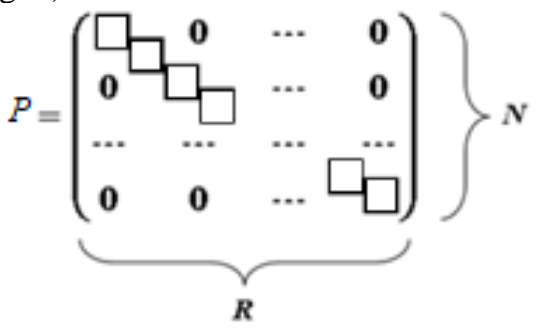

Fig. 3. Schematic matrix of planning

where squares are the Hermith cubic polynomials; $R-$ quantity of spline merging nodes on the monitoring interval.

For that let us find the web of nodes $\Delta_{R}$, on what the spline can be constructed. It is necessary to notice that web of nodes and the web of observations $\Delta_{\mathrm{N}}$ would be equal, in other words, several observations could be located on each section of the web of nodes that would define expected dependency behavior.

Each row for KEC consists of four basic functions, approximate form of which presented on the fig.4, that have a form of cubic polynomes that have been intersewed already under conditions of polynome steadiness and the steadiness of the first derivative in the merging node.

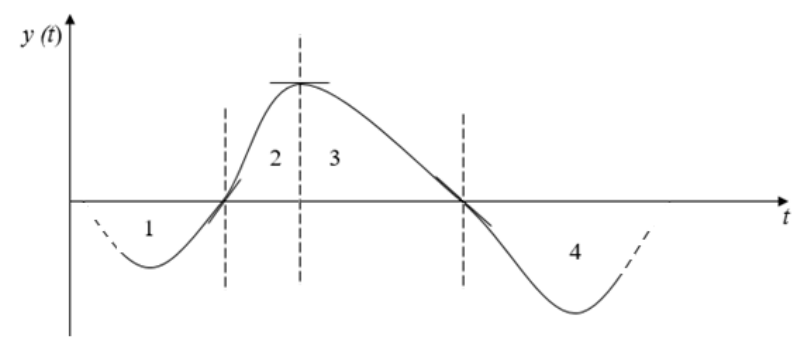

Fig. 4. Basis function presentation

Let us rewrite an expression (1) in the matrix form:

$$
[S p-Y]^{2}=\min
$$

where $Y$-input data vector.

$$
Y=\left[\begin{array}{c}
y_{1} \\
y_{2} \\
\vdots \\
y_{N}
\end{array}\right],
$$

where $y_{1}-y_{N}-$ input data value.

Let us write $S p$ spline in the following way:

$$
S p=P * A
$$

where $P$-planning matrix; $A$ - ordinate vector of the spline merging nodes.

For the whole KEC construction it is necessary to calculate ordinate vector of the spline merging nodes $A$, that has a form of:

$$
A=\left[\begin{array}{c}
a_{1} \\
a_{2} \\
\vdots \\
a_{R}
\end{array}\right] .
$$

where $a_{1}-a_{R}$ - ordinate values of the spline merging nodes.

The grade of nodes ordinate can be calculated by the least squares method, in other words their optimal values are constructing in content of maximal reasonableness.

Sought parameters can be founded by the way of matrix equations system solving. Taking into account an expression (3) the $A$ vector can be found:

$$
A=\left(P^{T} * P\right)^{-1} * P^{T} * Y
$$

Let us introduce following notations. Let us assume that

$$
\begin{aligned}
& C=P^{T} * P, \\
& B=P^{T} * Y .
\end{aligned}
$$

Including expressions (5) and (6), let us rewrite an expression (4) in the simplified form (4):

$$
A=C^{-1} * B
$$

where $C^{-1}$ - correlation fault matrix LSM.

Bring to notice that $C^{-1}$ is independent from the input counting and can be calculated preliminarily and weaved in the memory of the measuring device.

For the evaluation of the KEC usage let us accomplish modelling of the dependency between cellular network parameters KPI and KQI definition.

In the context of the work, the service of Internet access on the basis $3 \mathrm{G}$ cellular connection has been checked. For that purpose, the video file loading on the mobile phone from Internet has been accomplished (Fig. 5).

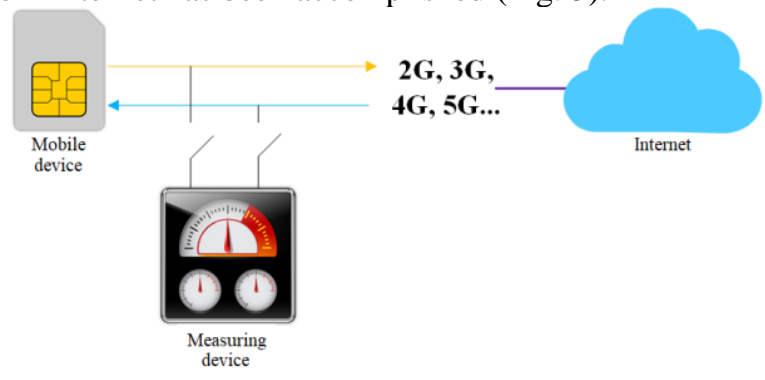

Fig. 5. Simplified network architecture 
KPI parameters were fixed on the measuring device: the speed of the input stream Y1, the speed of output stream Y2, delay Y3, signal power Y4. From the side of the user - KQI parameter - time of the Y5 video file loading.

On Fig. 6 the result of input stream speed data approximation by the Hermith cubic spline with spline merging node marks is represented.

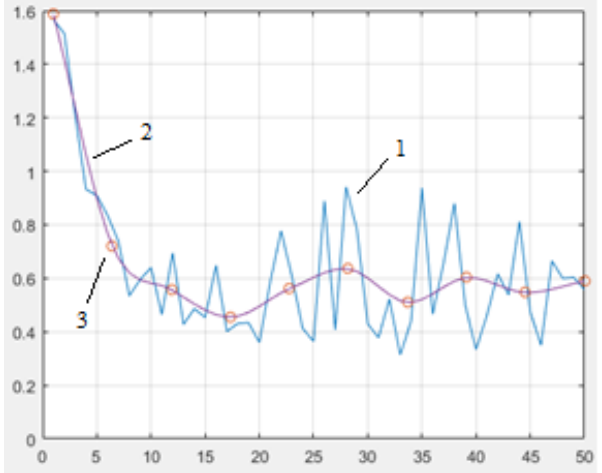

Fig. 6. Input stream speed approximation: 1 - curve of measured values; 2 - curve of approximated; 3 - merging nodes

Usage of splines allows to find not only statistical marks of spline-approximation sought parameters but also their confidence interval that increases certainty and probability of further calculations, so this undoubtedly is an advantage of the chosen approach. In our case it allows to receive the KQI forecast from KPI. At the perspective of development under such conditions the further step is the KPI concrete parameter choice realization in the aim of its improving for the increasing of the user service quality level.

\section{CONCLUSIONS}

In order to offer high quality services, it is not enough to simply collect all the information about customer preferences, it is also necessary to process such information in a timely manner to be able to act in real time. Increasing the amount of data to be processed in the architecture of the cellular provider increases the computation time and delays the workflow of the system. Current systems have the ability to collect all information and process it, but they are still unable to do so in a timely manner, unable to provide a good "management experience" to the end user.

Respective method based on the principles of machine learning has been developed to assess and optimize the state of the network in order to improve the quality of service provision to users by cellular operators using the model of service quality control system. Proposed method is a very effective instrument for request formation from users, network status monitoring, service providing quality control integral marks modelling and their optimization, search of degradation reasons and reporting ranging.

Also, the definition method of functional dependency between KPI and KQI on the basis of cubic Hermith splines usage has been performed in the development. This method allows to evaluate the influence rate of the each KPI parameter on KQI parameter with the future average data forecast that would tend to the realization of some KPI parameter choice for its further improvement and optimization for the aim of cellular provider service providing quality level increasing.

\section{ACKNOWLEDGEMENT}

This work was supported in part by the European Commission under the 5G-PPP project 5G-TOURS: SmarT mObility, media and e-health for toURists and citizenS (H2020-ICT-2018-2020 call, grant number 856950).

\section{REFERENCES}

[1] Steven Latr, Nicolas Staelens, Pieter Simoens. On-line estimation of the QoE of progressive download services in multimedia access networks [C]. ICOMP 2008, Las Vegas, Nevada, USA, 2008, pp. 117.

[2] Ricky K.P. Mok, Edmond W.W. Chan, and Rocky K.C. Chang. Inferring the QoE of HTTP Video Streaming from User-Viewing. Activities [C]. W-MUS [16] S. Mohamed and G. Rubino, "A Study of Real-time Packet Video Quality Using Random Neural Networks," IEEE Trans. On Circuits and Systems for Video Tech.,2002, 12(12), pp. 1071-1083

[3] Vaneet Aggarwal, Emir Halepovic. Prometheus: Toward Qua1ity-ofExperience Estimation for Mobile Apps from Passive Network Measurements, ACM HotMobile'14, Santa Barbara, CA, USA, February 26-27, 2014.

[4] Balachandran A., Sekar V., Akella A., Seshan S. et al. A quest for an Internet video Quality-of-Experience, metric. In ACM HotNets, 2012.

[5] Maxim Claeys. Design and Evaluation of a Self-Learning HTTP Adaptive Video Streaming Client, IEEE communications letters, Vol. 18. No. 4, April 2014

[6] VQEG, "Final report from the video quality experts' group on the validation of objective models of video quality assessment".

[7] Johan De Vriendt, Danny De Vleeschauwer. Model for estimating QoE of Video delivered using HTTP Adaptive Streaming [C], IFIP/IEEE IM, 2013:

[8] Goldshtein B., EvaginV., Belozertsev I. (2018). About quality of OTT Services in LTE. Vestnik Sviazy. 07, 7, pp. 9-12.

[9] Elagin V.S., Goldshtein A.B., Onufrienko A.V., Zarubin A.A., Belozertsev I.A. (2018). Synchronization of delay for OTT services in LTE. 2018 Systems of Signal Synchronization, Generating and Processing in Telecommunications (SYNCHROINFO), Minsk, 2018, pp. 1-4.

[10] Rules for providing and receiving telecommunication servicesl (Resolution of the Cabinet of Ministers of August 9, 2005 №720)

[11] ETSI TS 102 250-2 (2009-10) Speech Processing, Transmission and Quality Aspects (STQ); QoS aspects for popular services in GSM and 3G networks; Part 2: Definition of Quality of Service parameters and their computation (Аспекти оброблення мови, передавання та якості. Аспекти QoS популярних послуг, що надаються мережами GSM та 3G. Частина 2: Визначення показників якості послуг та їх розрахунок)

[12] Law of Ukraine "On Telecommunications", 18.11.2003p., N 1280-IV

[13] Huawei, Huawei eNodeB V100R005C00KPI, 2012.

[14] 3GPP Technical Specification 36201 v8.3.0, LTE Physical Layer general Description (Release 8) 2009

[15] 3GPP Technical Specification 32.450 v9.1.0, KPIs for E-UTRAN (Release 9) (2010)

[16] 3GPP Technical Spefication 32.454, KPI fo the IP Multimedia Subsystem (IMS) Definitions (Release 8) 2012

[17] Arzhanrsev S.V. Key mobile network performance indicators choise for customer experience estimation. T-Comm. 2016. Vol. 10. No. 6, pp. 69-72.

[18] "Vocabulary for performance and quality of service", 2006

[19] "The Open Group", October 2004.

[20] Shelevitsky I.V. Interpolation splines in digital signal processing problems. // Exponenta Pro. Mathematics in applications. 2003. -№4

[21] Shelevitsky I.V., Shutko M.O., Shutko V.M., Kolganova O.O. Splines in digital data and signal processing. 2007 - ISBN 078-9662915-86-0

[22] Malozemov V.N., Pevny A.B. Polynomial splines: Textbook. allowance. - Leningrad Publishing House. University, 1986 
